\title{
Hemodiafiltration for Intoxication with Lithium Unmasking Its Anti-Parkinsonian Effect
}

J osef Finsterer1, Claudia Stöllberger ${ }^{2 *}$ and Rebekka Wlassits ${ }^{2}$

${ }^{1}$ Rudolfstiftung Hospital, and the Messerli Institute, Vienna, Austria

${ }^{2}$ Department of Cardiology and Intensive Care Medicine, Rudolfstiftung Hospital, Vienna, Austria

*Correspondling author: Josef Finsterer, Rudolfstiftung Hospital, and the Messerli Institute, Vienna, Austria, Europe

Received: November 11, 2020; Accepted: January 19, 2021; Published: January 26, 2021

\section{Letter to the Editor}

From lithium it is known that it exhibits an anti-Parkinson effect [1]. Accordingly, smokers develop less frequently Parkinson's Disease (PD) than the non-smoking population [2]. This is because tobacco contains a significant amount of lithium [3]. The anti-PD effect of lithium is explained by increased autophagy and reduction of intracellular $\alpha$-synuclein [3]. Lithium inhibits GSK-3 $\beta$ and consequently increases the $\beta$-catenine activity [4]. Development of a Parkinson crisis due to flushing out of lithium by Hemodiafiltration (HDF), as in the following case, has not been reported.

The patient is a 63 years male (height $180 \mathrm{~cm}$, weight $75 \mathrm{~kg}$ ) who was living with his mother in a retirement home and developed fever since 4 days prior to admission. His previous history was positive for psychosis, bipolar disorder, arterial hypertension, chronic renal failure, chronic obstructive pulmonary disease, diabetes, bilateral hydrocele, prostate hypertrophy, polyneuropathy, lithium intoxication, and hyperlipidemia. He had the diagnosis of a questionable extra-pyramidal syndrome but no anti-Parkinson medication. His home medication included aripiprazole $(400 \mathrm{mg} /$ once every $30 \mathrm{~d})$, biperiden $(8 \mathrm{mg} / \mathrm{d})$, midazolam $(0.25 \mathrm{mg} / \mathrm{d})$, lithium $(900 \mathrm{mg} / \mathrm{d})$, quetiapine $(50 \mathrm{mg} / \mathrm{d})$, trazodone $(75 \mathrm{mg} / \mathrm{d})$, nebivolol $(2.5 \mathrm{mg} / \mathrm{d})$, metformin $(850 \mathrm{mg} / \mathrm{d})$, simvastatin $(20 \mathrm{mg} / \mathrm{d})$, tamsulosin $(0.4 \mathrm{mg} / \mathrm{d})$, fenoterol with ipratropium-bromid, and tiotropium plus olodaterol.

On admission (hospital day-1 (hd1)) he was comatose, had spontaneous resting tremor, and arterial hypotension (80/40 $\mathrm{mmHg}$ ). Blood tests revealed hypokaliemia, hypernatriemia, and renal insufficiency (Table 1). The swab test for SARS-CoV2 was negative. ECG showed AV-block-I, right bundle-branch-block, and QT-prolongation (501 ms). X-ray of the lung was normal. Clinical neurologic exam on hd2 revealed somnolence, pain upon maximal passive anteflexion of head, nuchal rigor, ptosis, ophthalmoparesis, spontaneous myocloni all over the body, mild rigor of all extremities, reduced tendon reflexes on the lower limbs, and clonus of the left lower leg (Table 1). Cerebral CT-scan was non-informative. He was treated with fluid substitution, cefuroxim, levetirazetam (LEV) (1000 $\mathrm{mg} / \mathrm{d}$ ), and discontinuation of all psychiatric medication.
Since EEG on hd3 showed a myoclonic sate, valproci acid (VPA) was added to LEV. Myocloni resolved but occasionally, generalized tonic-clonic seizures occurred, which responded to lorazepam. Since the lithium serum level was increased to $1.57 \mathrm{mmol} / \mathrm{L}$ ( $\mathrm{n}, 0.6 \mathrm{mmol} / \mathrm{L}$ to $1.0 \mathrm{mmol} / \mathrm{L}$ ) on hd3, lithium intoxication was diagnosed and HDF was begun. Under this regimen serum lithium levels successively became normal on from hd4 (Table 1). Clinical neurologic exam on hd4 surprisingly revealed an akinetic Parkinson crisis with massive, generalized rigor, inability to voluntarily move head or limbs or open the mouth despite discontinuation of all neuroleptic drugs (Table 1). L-DOPA had a beneficial effect. The patient could be discharged without seizures, PD manifestations, or electrolyte disturbances on hd36. Lithium remained discontinued.

The presented patient is educative for several aspects. First, lithium had obviously an anti-Parkinson effect, as lowering the serum lithium level by HDF induced a massive PD crisis. Second, lithium intoxication manifested with coma, resting tremor, hyperthermia, lower-leg clonus, generalized myocloni, and generalized tonicclonic seizures. Third, discontinuation of psychiatric medication and application of L-DOPA resulted in complete resolution of the Parkinson crisis. Whether ptosis and ophthalmoparesis were due to myopathy or myasthenia, triggered by the lithium intoxication, as

Table 1: Findings on clinical neurologic examination and laboratory investigations before and after HDF.

\begin{tabular}{|c|c|c|}
\hline \\
\hline & Before HDF (hd2) & After HDF (hd4) \\
\hline Consciousness & comatose & somnolence \\
\hline Myocloni & generalized & none \\
\hline GTCS & yes & none \\
\hline Clonus & left lower leg & none \\
\hline PD & mild & severe \\
\hline Opening of mouth & possible & impossible \\
\hline Neck rigor & mild & severe (inability to move head) \\
\hline Rigor of limbs & mild & severe \\
\hline Hypokinesia & mild & severe \\
\hline Resting tremor & yes & yes \\
\hline Reduced DTR & yes & yes \\
\hline Ptosis & yes & yes \\
\hline Ophthalmoparesis & yes & yes \\
\hline EEG & myoclonic state & no paroxysmal activity \\
\hline Potassium (3.4-4.5mmol/l) & $4.9 \mathrm{mmol} / \mathrm{l}$ & $3.8 \mathrm{mmol} / \mathrm{l}$ \\
\hline Sodium $(136-145 \mathrm{mmol} / \mathrm{l})$ & $150 \mathrm{mmol} / \mathrm{l}$ & $145 \mathrm{mmol} / \mathrm{l}$ \\
\hline Creatinine (0-7-1.2mg/dL) & $1.8 \mathrm{mg} / \mathrm{dL}$ & $0.68 \mathrm{mg} / \mathrm{dL}$ \\
\hline Lithium $(0-6-1.0 \mathrm{mmol} / \mathrm{L})$ & $1.57 \mathrm{mmol} / \mathrm{L}$ & $0.74 \mathrm{mmol} / \mathrm{L}$ \\
\hline
\end{tabular}

GTCS: Generalized Tonic-Clonic Seizures; PD: Parkinson's Disease; DTR: Deep Tendon Reflexes
J Fam Med - Volume 8 Issue 1 - 2021

ISSN : 2380-0658 | www.austinpublishing group.com

Stöllberger et al. (C) All rights are reserved
Citation: Finsterer J, Stöllberger C and Wlassits R. Hemodiafiltration for Intoxication with Lithium Unmasking Its Anti-Parkinsonian Effect. J Fam Med. 2021; 8(1): 1237. 
previously reported [5], remains speculative.

Keywords: Lithium; Hemodiafiltration; Parkinsonism; Psychosis; Extra-pyramidal symptoms

\section{Author Contribution}

JF: design, literature search, discussion, first draft, critical comments: literature search, critical comments, revision.

\section{References}

1. Lieu CA, Dewey CM, Chinta SJ, Rane A, Rajagopalan S, Batir S, et al. Lithium prevents parkinsonian behavioral and striatal phenotypes in an aged parkin mutant transgenic mouse model. Brain Res. 2014; 1591: 111-117.
2. Dick FD, De Palma G, Ahmadi A, Scott NW, Prescott GJ, Bennett J, et al. Environmental risk factors for Parkinson's disease and parkinsonism: the Geoparkinson study. Occup Environ Med. 2007; 64: 666-672.

3. Guttuso T. High lithium levels in tobacco may account for reduced incidences of both Parkinson's disease and melanoma in smokers through enhanced $\beta$-catenin-mediated activity. Med Hypotheses. 2019; 131: 109302.

4. Kurgan N, Whitley KC, Maddalena LA, Moradi F, Stoikos J, Hamstra SI, et al. A low-therapeutic dose of lithium inhibits GSK3 and enhances myoblast fusion in $\mathrm{C}_{2} \mathrm{C}_{12}$ cells. Cells. 2019; 8: 1340.

5. Ronziere T, Auzou P, Ozsancak C, Magnier P, Senant J, Hannequin D. Myasthenic syndrome induced by lithium. Presse Med. 2000; 29: 1043-1044. 